\title{
Distribución y patrones espaciales del impacto de un ingeniero de los ecosistemas exótico e invasor, Castor canadensis, en Tierra del Fuego, Argentina
}

\author{
Ana Eljalli; Hernán Dieguez ${ }^{12}{ }^{12}$; M. Fernanda Menvielle ${ }^{3}$ \& Karina Hodara ${ }^{1}$ \\ ${ }^{1}$ Departamento de Métodos Cuantitativos y Sistemas de Información, Facultad de Agronomía, Universidad de Buenos Aires \\ (FAUBA), Buenos Aires, Argentina. ${ }^{2}$ Laboratorio de Análisis Regional y Teledetección, IFEVA (CONICET-FAUBA), Buenos \\ Aires, Argentina. ${ }^{3}$ Administración de Parques Nacionales, Buenos Aires, Argentina.
}

\begin{abstract}
RESUMEN. El castor americano (Castor canadensis) es una especie ingeniera de los ecosistemas introducida en 1946 en Tierra del Fuego, en el extremo austral de América del Sur. En la actualidad, está presente en los diferentes ambientes del archipiélago fueguino, donde transforma notablemente la estructura y el funcionamiento de los ecosistemas, en particular al inundar ambientes riparios mediante la construcción de diques. El objetivo de este trabajo fue determinar, por primera vez a partir de un relevamiento exhaustivo, los patrones espaciales de la distribución y el número de diques construidos por esta especie en la porción argentina de la Isla Grande de Tierra del Fuego. Para esto se elaboró una base de datos geográfica con el registro de diques mediante la interpretación visual de imágenes satelitales de acceso libre y de alta resolución espacial. Se identificaron 70682 diques, cuya longitud acumulada supera $\operatorname{los} 2300 \mathrm{~km}$ y la superficie que inundan es de, al menos, 87 $\mathrm{km}^{2}$. La mayor densidad de diques se observó en la región cordillerana, donde alcanzó valores máximos de 123 diques $/ \mathrm{km}^{2}$ al SE del lago Fagnano. En la porción sur de la isla, el 84\% del territorio se encuentra a menos de $1 \mathrm{~km}$ de un dique. La información generada en este trabajo es de uso libre para fines de investigación y divulgación. Se la podrá usar para poner a prueba hipótesis sobre los controles biofísicos y humanos de la invasión, para identificar áreas prioritarias para investigación y desarrollar estrategias de manejo, así como para monitorear la invasión en el espacio y en el tiempo, detectar tempranamente nuevas áreas invadidas y entrenar métodos automáticos de detección de áreas impactadas por el castor.
\end{abstract}

[Palabras clave: invasión biológica, mamíferos exóticos, Patagonia, SIG, Google Earth]

\begin{abstract}
Distribution and spatial patterns of the impacts of an exotic and invasive ecosystem engineer: Castor canadensis in Tierra del Fuego, Argentina. The American beaver (Castor canadensis) is an ecosystem engineer introduced in 1946 in Tierra del Fuego, in the southernmost end of South America. Currently, beavers inhabit the different environments of the Fuegian archipelago, where they generate significant changes in the structure and functioning of ecosystems, mainly through the flooding of riparian areas by means of dam construction. The aim of this work was to describe, for the first time through an exhaustive survey, the spatial patterns of the distribution and density of beaver dams in the Argentinian portion of the Isla Grande de Tierra del Fuego. With this purpose, a geographic database was created, registering dams by means of visual interpretation of freely accessible satellite images with high spatial resolution. A total of 70682 dams were identified whose cumulative length exceeds $2300 \mathrm{~km}$ and flooded an area of at least $87 \mathrm{~km}^{2}$. The highest dam density was observed in the cordilleran region, reaching maximum values of up to $123 \mathrm{dams} / \mathrm{km}^{2}$ in the SE of Fagnano lake. In the southern portion of the island, $84 \%$ of the territory is less than $1 \mathrm{~km}$ away from its nearest beaver dam. The information generated in this work is free to use for research purposes. It can be used to test hypotheses about the biophysical and human controls of the invasion, the identification of priority areas for research and the development of management strategies, as well as for the monitoring of the invasion in space and time, the early detection of new invaded areas and the training of automatic methods for the detection of areas impacted by the beaver.
\end{abstract}

[Keywords: biological invasion, exotic mammals, Patagonia, GIS, Google Earth]

\section{INTRODUCCIÓN}

Las invasiones biológicas son consideradas una dimensión del cambio global, tan importante como los cambios climáticos, la alteración de la composición atmosférica y los cambios en el uso y la cobertura del suelo, debido a la amenaza que ejercen sobre la conservación de la biodiversidad y la provisión de servicios ecosistémicos (Vitousek et al. 1996;

Editor asociado: Fernando Milesi
Chapin et al. 2000; Mack et al. 2000; Ehrenfeld 2010). Conocer la distribución y abundancia de las especies invasoras permite poner a prueba hipótesis sobre los controles biofísicos y humanos de la invasión, identificar áreas prioritarias para investigación y desarrollar estrategias de manejo. También habilita el monitoreo de la invasión en el espacio y en el tiempo, y la detección temprana de nuevas áreas invadidas. En particular, es un insumo

Recibido: 5 de Abril de 2018

Aceptado: 19 de Septiembre de 2018 
fundamental para diseñar un manejo efectivo y emprender la restauración de las áreas afectadas, en los casos en que sea posible.

Los ambientes insulares suelen ser más susceptibles a las invasiones y a sus consecuencias (Courchamp et al. 2003; Tershy et al. 2015; Bellard et al. 2016). En Tierra del Fuego, un archipiélago situado en el extremo austral del continente americano, al menos 24 especies de vertebrados exóticos tienen poblaciones establecidas (Valenzuela et al. 2014). Entre ellas, el castor americano (Castor canadensis) es una de las más ampliamente distribuidas, y su impacto es notable. Debido a su expansión exitosa y alarmante, que incluso alcanza a la Patagonia continental (Pietrek and Fasola 2014; Graells et al. 2015), los gobiernos de la Argentina y de Chile firmaron en 2008 el "Acuerdo Sobre la Restauración de los Ecosistemas Australes Afectados por el Castor Canadiense (Castor canadensis)", mediante el que ambos países se plantean erradicar la especie en todo su rango de distribución (Malmierca et al. 2011; Schiavini et al. 2016).

El castor es un roedor semiacuático, herbívoro y generalista, nativo de América del Norte e invasor en el extremo sur de Sudamérica. En el año 1946 se introdujeron 20 ejemplares de esta especie cerca de la desembocadura del río Claro en el lago Fagnano, en la porción argentina de la Isla Grande de Tierra del Fuego, con el objetivo de estimular la industria peletera (Pietrek and Fasola 2014). Desde su introducción, el castor se expandió por el archipiélago fueguino, donde llegó a ocupar el 98\% de las cuencas hídricas del territorio (Lizarralde et al. 2008). El éxito de su expansión en tan pocas décadas se debería a la escasez de competidores y depredadores naturales, a la abundante disponibilidad de recursos, a la gran plasticidad característica de la especie y a la semejanza fisonómica y climática entre los ambientes invadidos y los nativos (Wallem et al. 2007).

El castor es considerado una especie ingeniera de los ecosistemas debido a la diversidad y a la magnitud de los impactos que genera en los sistemas ribereños (Naiman et al. 1988; Jones et al. 1994; Wright et al. 2002; Valenzuela et al. 2014). Construye diques que cortan el flujo de agua de los ríos y arroyos, lo que genera embalses y altera la composición y el funcionamiento ecosistémico del ambiente donde se establece. Ensancha el cauce de los ríos, lo cual altera su morfología y las dinámicas hídrica y ecológica (Lizarralde et al. 2004), y degrada los bosques ribereños tanto por efecto de la inundación como por el corte o anillamiento de los árboles utilizados para construir las represas y alimentarse (Anderson et al. 2006; Martínez Pastur et al. 2006). Estos cambios en el ambiente facilitarían su invasión por otras especies exóticas (Anderson et al. 2006; Martínez Pastur et al. 2006; Moorman et al. 2009; Valenzuela et al. 2014).

Las imágenes aéreas o satelitales de alta resolución espacial, es decir con un tamaño de píxel de unos pocos metros de lado, son una herramienta muy útil para estudiar las invasiones biológicas a escala regional (Visser et al. 2013). De las imágenes de alta resolución se puede obtener información tanto de la población objetivo como de sus hábitats y, en algunos casos, es posible estimar densidades poblacionales y tasas de crecimiento poblacional, además de evaluar el uso del ambiente y la tasa de dispersión de la especie blanco (Fretwell et al. 2012; Visser et al. 2013). Google Earth y Bing Maps son plataformas que proveen acceso gratuito a este tipo de imágenes de toda la superficie del planeta, de distintos momentos, actualizadas de forma regular y a través de una interfaz de uso sencillo para los usuarios (Butler 2006; Yu et al. 2012). Los cambios en el paisaje producidos por la actividad del castor pueden ser detectados a través del análisis de imágenes satelitales y aéreas. De hecho, la interpretación de fotografías aéreas o imágenes satelitales identificando diques como indicador de la presencia de castor, aunque no se pueda conocer la ocupación actual efectiva de cada uno de ellos, fue utilizada con éxito en estudios recientes en Tierra del Fuego (Pietrek and González-Roglich 2015; Henn et al. 2016) y en el hemisferio norte (Johnston and Naiman 1990; Butler 2012; Morrison et al. 2014; Martin et al. 2015).

Hasta el momento, no existe información cuantitativa actualizada y espacialmente explícita de la distribución y la abundancia de la población de castor, con una resolución adecuada a los fines de manejo; es decir, que abarque una extensión espacial amplia y con un nivel de detalle que permita identificar con precisión los sitios impactados por el castor. En la porción chilena de la Isla Grande de Tierra del Fuego existen caracterizaciones cualitativas de la distribución espacial y de los patrones de colonización del castor (Briones et al. 2001; Skewes et al. 2006). Por su parte, la porción argentina de la isla cuenta con estudios cuantitativos que abarcan una 
extensión espacial limitada (Coronato et al. 2003; Pietrek and González-Roglich 2015) o se basan en muestreos para la caracterización a nivel regional (Henn et al. 2016) y no en un relevamiento exhaustivo de todo el territorio. En este trabajo se describen los patrones espaciales de la distribución y el número de diques del castor americano en la porción argentina de la Isla Grande de Tierra del Fuego y se cuantifican algunos de sus impactos. Los objetivos específicos fueron: 1) generar una base de datos a partir de la identificación de signos de presencia de castor sobre imágenes de alta resolución espacial, 2) cartografiar la distribución y el número de diques construidos por castores, 3) estimar métricas cuantitativas de la presencia e impacto del castor y 4) contrastar la cartografía elaborada con información relevada en el terreno. La información generada en este trabajo es de acceso libre para usos científicos, de divulgación y de manejo de la especie.

\section{Materiales y MÉTODOS}

\section{Área de estudio}

La porción argentina de la Isla Grande de Tierra del Fuego abarca una superficie de $\sim 21000 \mathrm{~km}^{2}$. Está ubicada en el extremo austral del continente americano, al este del meridiano $68^{\circ} 36^{\prime} \mathrm{O}$ y entre los paralelos $52^{\circ} 39^{\prime}$ y $55^{\circ} 4^{\prime} \mathrm{S}$. Según la clasificación de Köppen (1936) y datos del Servicio Meteorológico Nacional (SMN 2018), el clima es subpolar oceánico, con temperatura media superior a $10{ }^{\circ} \mathrm{C}$ en menos de cuatro meses al año y oscilación anual escasa. Las precipitaciones varían entre los 300 y $1500 \mathrm{~mm} /$ año, sin estación seca, y presentan un incremento marcado de norte a sur y a medida que aumenta la altura sobre el nivel del mar. Una buena parte de las precipitaciones invernales cae en forma de nieve, que se acumula de manera permanente sobre los $700 \mathrm{~m} \mathrm{s.n.m}$.

El territorio fueguino se puede dividir en cuatro regiones ecológicas considerando la vegetación dominante y siguiendo los límites de las cuencas hidrológicas: Norte, Ecotono, Cordillera y Península Mitre (Iturraspe and Urciolo 2000). En el norte de la isla se distribuye la estepa magallánica, cuya comunidad característica está dominada por Festuca gracillima. La topografía determina la presencia de pampas cubiertas por coironales de $F$. gracillima en tierras altas, y de vegas o mallines en el fondo de valles y cañadones, donde la napa freática se encuentra cerca de la superficie. Las vegas están cubiertas por comunidades herbáceas higrofíticas de gramíneas y ciperáceas dominadas por Hordeum comosum y Carex canescens. En la zona central de la isla se encuentra un amplio ecotono entre las estepas del norte y los bosques del sur. El paisaje está constituido por un mosaico de islas de bosque en las zonas más altas rodeado por estepas y praderas en las partes bajas. La especie arbórea predominante es Nothofagus antarctica (ñire), que presenta una altura no superior a los $12 \mathrm{~m}$, forma achaparrada, gran plasticidad y ausencia de hojas durante el otoño-invierno. En el SO, la topografía es montañosa y se caracteriza por la alternancia de grandes sierras y valles de modelado glacial. Predominan los bosques deciduos dominados por Nothofagus pumilio (lenga), que también coexiste en bosques mixtos con la especie perennifolia N. betuloides (guindo o coigüe de Magallanes) en zonas más húmedas como los litorales costeros o bordes de lagos, y con $N$. antarctica en zonas más secas. Nothofagus betuloides también forma bosques puros en parches de mediana extensión. Los bosques forman un mosaico con los turbales que aparecen en los valles y zonas donde la napa freática llega a la superficie. A mayor altitud, la vegetación de montaña está constituida por formaciones herbáceas y de arbustos rastreros como Empetrum rubrum o arbustos en cojín como Bolax gummifera. Al SE de la isla se encuentra la Península Mitre, dominada por turbales del musgo Sphagnum magellanicum, asociado frecuentemente con Empetrum rubrum. También hay turbales con asociación de Donatia fascicularis y Astelia pumila y en las partes más altas del paisaje se encuentran bosques de Nothofagus (Pisano 1977; Moore 1983).

\section{Metodología}

Se registraron signos de presencia del castor a partir de la interpretación visual de imágenes satelitales obtenidas entre 2002 y 2016, relevando exhaustivamente toda la superficie de la porción argentina de la Isla Grande de Tierra del Fuego. Los signos de utilización del hábitat por parte de esta especie se identifican con facilidad en fotografías aéreas y en imágenes de alta resolución (Pietrek and González-Roglich 2015; Henn et al. 2016). En este caso, los signos digitalizados fueron diques construidos con o sin embalse asociado, destrucción del bosque ribereño, y presencia de canales y madrigueras en los cuerpos de agua (Figura 1). 


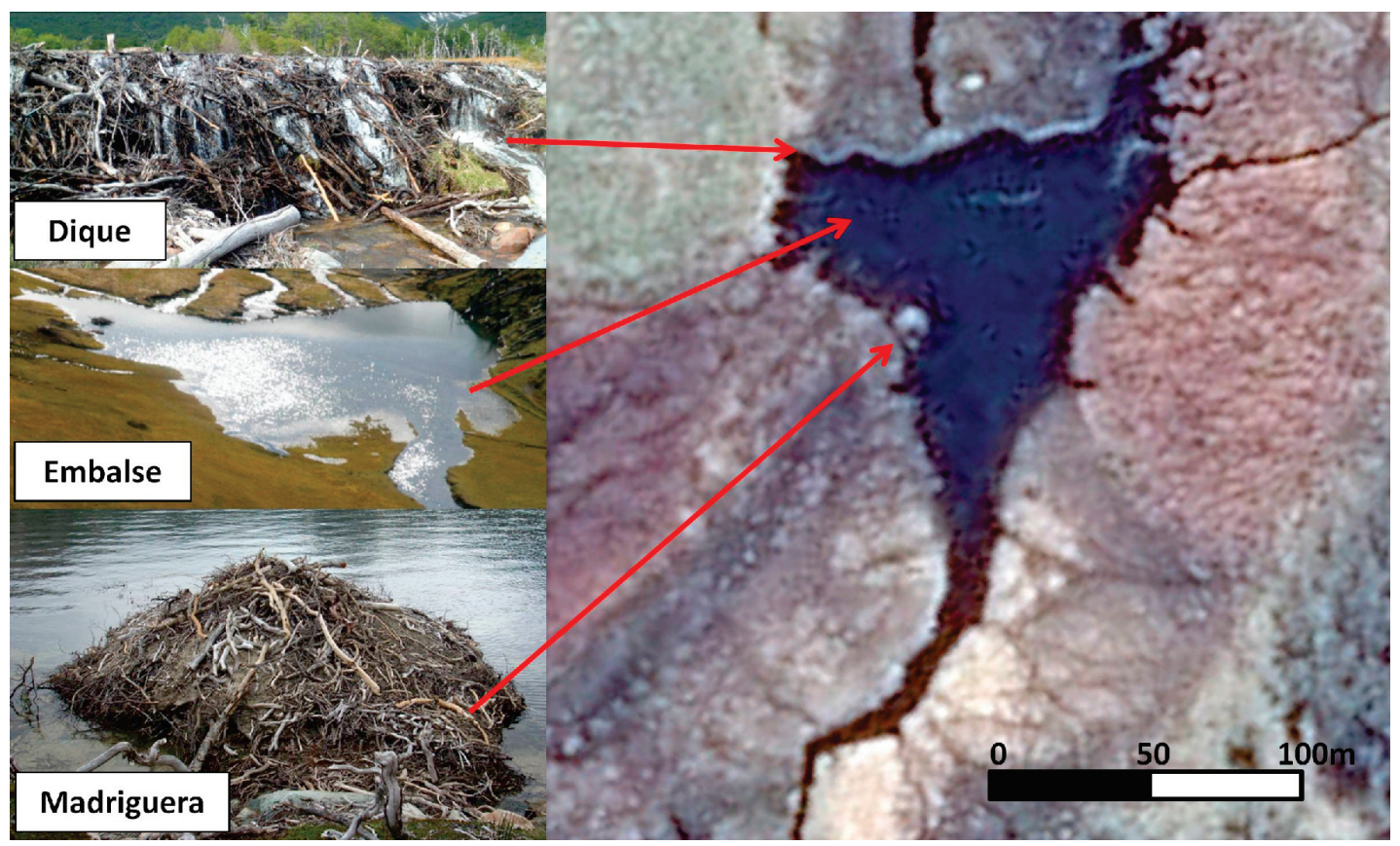

Figura 1. Fotointerpretación de diques, embalses y madrigueras de castor. A la izquierda se muestran fotografías de cada uno de estos elementos y a la derecha una imagen satelital de alta resolución espacial.

Figure 1. Visual interpretation of beaver dams, ponds and lodges. On the left, photographs of each of these elements and on the right, a high spatial resolution satellite image.

La fotointerpretación se realizó durante 2016 y 2017 a partir de las imágenes más actuales disponibles en la plataforma Google Earth en ese momento. En aquellos sitios donde las imágenes disponibles en esta plataforma eran de baja calidad (debido a presencia de nubes o a baja resolución espacial), el relevamiento se complementó con imágenes de Bing Maps. A partir de esta información se generó una base de datos espacialmente explícita. Luego, se cartografió la distribución espacial y la densidad de diques como indicador de la presencia del castor (Pietrek and GonzálezRoglich 2015; Henn et al. 2016), y se usó como base una grilla hexagonal con un tamaño de celda de $1 \mathrm{~km}^{2}$.

La superficie inundada se cuantificó mediante un muestreo aleatorio de diques, estratificado por región ecológica, y a partir de la digitalización de los embalses asociados a los mismos. Para esto se realizó un muestreo piloto relevando 80 diques en cada región y se estimó el tamaño óptimo de muestra por región de acuerdo a la metodología propuesta por Krebs (1989). Esta metodología considera la variabilidad entre unidades y un cierto error aceptado alrededor de la media para determinar un número confiable y definitivo de unidades de muestreo. Se estableció un error máximo de $15 \%$ alrededor del promedio, que determinó que el número de embalses a relevar fueran 294 en la región Norte, 311 en Ecotono, 239 en Cordillera y 339 en Península Mitre.

Por último, se realizó una evaluación cuantitativa de la capacidad de detección de diques mediante interpretación visual a partir de la comparación con datos relevados en el terreno. Para ello se utilizaron 87 sitios con presencia de castor relevados en las regiones Norte y Cordillera (Pietrek et al. 2017). Se calculó la cantidad de coincidencias entre la presencia de castor relevada en el terreno y la presencia de diques digitalizados en la base de datos generada. En estos sitios, las imágenes satelitales examinadas fueron del mismo año o posteriores al relevamiento de los datos registrados a campo.

\section{Resultados}

Mediante el relevamiento exhaustivo de imágenes satelitales se digitalizaron 72547 signos de presencia del castor, de los cuales 70682 correspondieron a diques y 1865 a otros signos como la afectación de la estructura del bosque o la presencia de madrigueras en cuerpos de agua. Esta información se usó como base para la generación de un mapa de alta resolución espacial que muestra la extensión y 


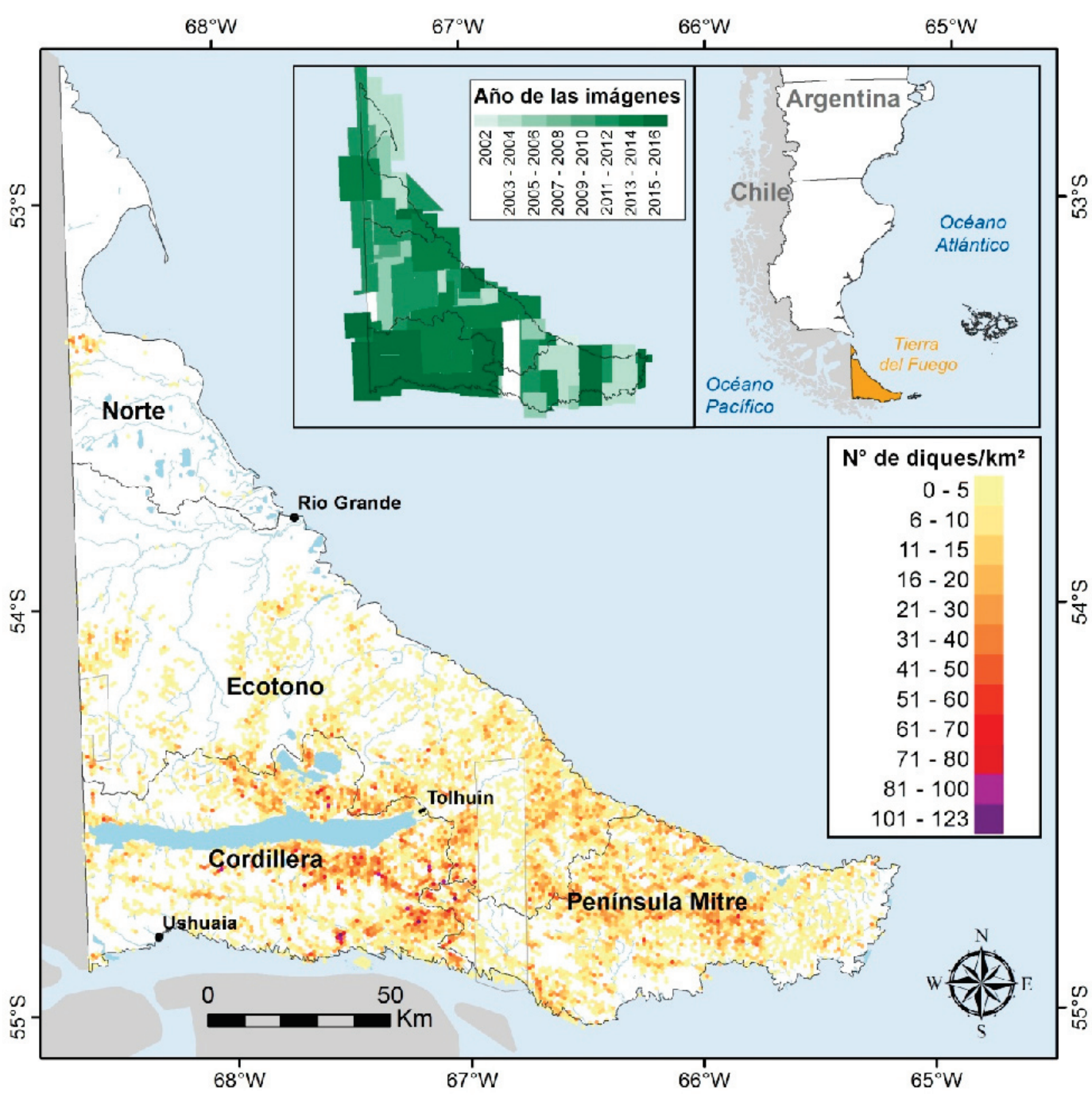

Figura 2. Densidad de diques construidos por castores en la porción argentina de la Isla Grande de Tierra del Fuego. Los recuadros grises (áreas blancas en el mapa inserto) indican zonas donde la baja resolución de las imágenes satelitales o la presencia de nubes dificultaron la identificación de diques.

Figure 2. Beaver dams density in the Argentinian portion of the Isla Grande de Tierra del Fuego. Grey polygons (white areas in the inset) show sites where low resolution and/or clouds in the satellite images hindered the proper identification of dams.

Tabla 1. Densidad de diques y superficie inundada por región y agregado para toda la extensión de la porción argentina de la Isla Grande de Tierra del Fuego según la fotointerpretación de imágenes satelitales de alta resolución

Table 1. Density of dams and flooded area by region and aggregated for the Argentinian portion of the Isla Grande de Tierra del Fuego, according to the visual interpretation of high spatial resolution satellite imagery.

\begin{tabular}{lccccc}
\hline Región & $\begin{array}{c}\text { Superficie } \\
\left(\mathrm{km}^{2}\right)\end{array}$ & $\begin{array}{c}\text { Número de } \\
\text { diques }\end{array}$ & $\begin{array}{c}\text { Densidad } \\
\text { media } \\
\left(\text { diques } / \mathrm{km}^{2}\right)\end{array}$ & $\begin{array}{c}\text { Densidad } \\
\text { máxima } \\
\left(\text { diques } / \mathrm{km}^{2}\right)\end{array}$ & $\begin{array}{c}\text { Superficie } \\
\text { inundada } \\
\left(\mathrm{km}^{2}\right)\end{array}$ \\
\hline Norte & 2522 & 457 & 0.18 & 36 & 0.3 \\
Ecotono & 8525 & 20810 & 2.44 & 76 & 25.8 \\
Cordillera & 4785 & 26139 & 5.46 & 123 & 40.14 \\
Península Mitre & 3935 & 23276 & 5.92 & 101 & 20.97 \\
Isla Grande (Argentina) & 19767 & 70682 & 3.39 & 123 & 87.21 \\
\hline
\end{tabular}


los patrones espaciales de la invasión (Figura 2; Apéndice 1). La mayor cantidad de diques se observó al sur de la Isla Grande de Tierra del Fuego, en la frontera entre las regiones Cordillera, Ecotono y Península Mitre, con una densidad máxima de 123 diques $/ \mathrm{km}^{2}$. La densidad media más alta $\left(5.92\right.$ diques $/ \mathrm{km}^{2}$ ) corresponde a la Península Mitre, seguida por la Cordillera (5.46 diques $/ \mathrm{km}^{2}$ ); en el Ecotono se registraron valores intermedios de 2.44 diques $/ \mathrm{km}^{2}$, y en la región Norte se registró la menor densidad media de diques, con 0.18 diques $/ \mathrm{km}^{2}$ (Tabla 1). En la porción sur de la isla (Cordillera y Península Mitre), $8 \%$ del territorio se encuentra a menos de 100 $\mathrm{m}$ de un dique, mientras que $84 \%$ del territorio se encuentra a menos de $1 \mathrm{~km}$ del dique más cercano.

La longitud acumulada para el total de diques digitalizados en el área de estudio fue $2324 \mathrm{~km}$. Estos diques generan una superficie inundada en los embalses asociados estimada en 87.21 $\mathrm{km}^{2}$, equivalente a $0.4 \%$ de la superficie del área de estudio. La región con más superficie total inundada fue la Cordillera, seguida por el Ecotono, la Península Mitre y, finalmente, la región Norte, mientras que, en términos relativos a su superficie, el orden decreciente es Cordillera, Península Mitre, Ecotono y Norte (Tabla 1).

Al comparar los diques relevados mediante fotointerpretación con la presencia de castor verificada en el terreno se observó un $85 \%$ de coincidencias en la región norte y un 77\% en la zona cordillerana. Las discrepancias estuvieron asociadas principalmente a dos tipos de situaciones. Por un lado, a la presencia de castores en sitios como pequeñas lagunas, en donde no necesitan construir diques para manejar el flujo de agua. Por otro lado, durante el relevamiento terrestre se encontraron pequeños diques ubicados bajo el dosel arbóreo en zonas boscosas donde el impacto de la especie aún no es suficiente para generar claros en la vegetación; en esos casos, la fotointerpretación a partir de imágenes satelitales se ve imposibilitada.

\section{DisCUSIÓN}

Este trabajo aporta información actualizada y espacialmente explícita sobre el estado de la invasión del castor en la porción argentina de la Isla Grande de Tierra del Fuego. Mediante el relevamientoexhaustivoy la fotointerpretación de imágenes satelitales se digitalizaron de forma manual más de 70000 diques. Con ellos se generó un mapa que revela la amplia distribución y la intensidad de una invasión biológica exitosa y con impactos notables: una longitud acumulada de diques que supera los $2300 \mathrm{~km}$, dos veces el perímetro del área de estudio, y la inundación de al menos 87 $\mathrm{km}^{2}$, una superficie equivalente a la de 11600 canchas de fútbol. Más aun, en la porción sur de la isla (Cordillera y Península Mitre), $84 \%$ de la superficie se encuentra a una distancia menor a $1 \mathrm{~km}$ del dique más cercano, mientras que $16 \%$ restante corresponde mayoritariamente a las altas cumbres y a grandes cuerpos de agua. La evaluación realizada con datos tomados en el terreno evidencia un elevado nivel de precisión para identificar áreas impactadas por castores. La importancia de disponer de herramientas para la detección temprana de áreas invadidas se hace evidente, ya que la especie tiene el potencial de expandir su distribución; ya alcanzó el continente y se confirmó su presencia en sitios tan al norte como Puerto Natales, Chile (Pietrek and Fasola 2014; Graells et al. 2015).

Nuestros resultados son congruentes con los de otros trabajos realizados en Tierra del Fuego. Lizarralde (1993) también observó que la densidad de diques era más alta en la Península Mitre, seguida por ambientes donde predominaba el bosque. En el trabajo realizado por Skewes et al. (2006) se registró el mismo tipo de gradiente de abundancia creciente norte-sur en la porción chilena de la isla, que pudimos verificar en la porción argentina y que está asociado a un incremento de la superficie boscosa y de la densidad de la red hidrográfica. La superficie inundada debido a la construcción de diques estimada en este trabajo es similar a la reportada por Henn et al. (2016), también para la porción argentina de la Isla Grande de Tierra del Fuego, quienes a su vez informaron una subestimación del área afectada en 13\%. Si bien el corte de la vegetación por los castores abarca una mayor superficie (Henn et al. 2016), se considera que la inundación genera más impacto sobre la estructura del bosque que la remoción debido a que el anegamiento ocasiona la pérdida de todo el bosque ribereño (Baldini et al. 2008; Hyvönen and Numi 2008). Ese impacto perdura aun después de que los embalses son abandonados y drenados, dado que la acumulación de sedimentos dificulta la regeneración de especies leñosas nativas como Nothofagus pumilio o N. betuloides (Anderson et al. 2006; Martínez Pastur et al. 2006). 
Los modelos que predicen la presencia del castor en el área de estudio indican que la disponibilidad de agua, de forraje y la topografía son las variables que determinan significativamente la invasión y el establecimiento de castores en los diferentes ambientes (Henn et al. 2016). La mayor densidad de diques se observó en el este de la región Cordillera, el sur del Ecotono y el oeste de la Península Mitre, sitios que presentan una matriz boscosa, una red de drenaje abundante y más tiempo de invasión (Pietrek and Fasola 2014). Sin embargo, la distribución del castor no se restringe a estos sitios; la especie también está presente, aunque con mucha menor densidad, en zonas donde no hay árboles y los drenajes son poco densos (Skewes et al. 2006; Pietrek and González-Roglich 2015; Henn et al. 2016). En la región Cordillera, los diques se encuentran mayormente en los fondos de los valles, pero también en las laderas de las montañas (Coronato et al. 2003). En Península Mitre, es posible que la densidad elevada de diques esté asociada a la gran capacidad de retención de humedad en las turberas (Turetsky and St. Louis 2006; Morrison et al. 2014), sumado a la presencia de bosque y de numerosas lagunas. El Ecotono es una región de transición donde los ambientes más impactados por el castor son los turbales y el bosque (Henn et al. 2016). En la región Norte se registró la menor presencia del castor, que ocupa sectores aislados. En este ambiente, el más recientemente invadido, la red de drenaje es poco densa y los reservorios de agua son limitados (Iturraspe and Urciuolo 2000). La presencia del castor en la estepa, como la construcción de embalses en arroyos temporarios, sugiere que el escaso caudal no es un factor limitante (Coronato et al. 2003). Más aun, trabajos recientes documentaron la capacidad que posee la especie de manejar el agua en ausencia de material leñoso (Pietrek and González-Roglich 2015). Aunque en esta región se registró un número de diques más bajo, posiblemente debido a un hábitat de menor calidad (aunque no limitante) (Skewes et al. 2006), se resalta la necesidad de tenerla en consideración como una potencial fuente de propágulos hacia la Patagonia continental (Pietrek et al. 2017).

Los resultados de este trabajo no permiten realizar estimaciones poblacionales ni se debe interpretar que todos los embalses están actualmente ocupados. Las colonias de castores tienen un número variable de individuos y, a su vez, cada colonia puede ocupar un número variable de embalses (Baker and Hill 2003; Lizarralde et al. 2004). A su vez, se reportó la existencia de castores que no construyen diques y habitan en lagos o lagunas, en remansos de ríos meandrosos e incluso en ríos caudalosos (Schiavini, comunicación personal). Por otro lado, mediante la fotointerpretación no es posible inferir la ocupación real de cada embalse. Los diques construidos por los castores perduran en el tiempo y se pueden identificar a través de imágenes satelitales muchos años después de ser abandonados.

La actividad del castor genera un patrón observable en imágenes satelitales. Este patrón es muy diferente al de otros factores que pueden afectar al bosque o a los cursos de agua. Debido a esto, afirmamos con suficiente confianza que, entre los registros de nuestra base de datos, la cantidad de falsos positivos (i.e., sitios que parecen afectados, pero sin presencia actual o pasada de castores) es mínima. Sin embargo, el uso de imágenes satelitales presenta algunas restricciones para detectar la presencia del castor $y$, por lo tanto, existen casos de falsos negativos (i.e., sitios con presencia actual o reciente de castores, no incluidos en nuestra base de datos). La presencia de nubes y la baja resolución espacial de algunas imágenes satelitales en sectores acotados (indicados con líneas grises en la Figura 2), así como también las sombras de los árboles y los troncos caídos sobre los embalses pueden dificultar la fotointerpretación. Además, en los bosques, los diques no pueden ser detectados hasta que el impacto de la actividad del castor produce la apertura del dosel. Otra de las limitaciones de nuestros resultados es que, en algunos sectores, las imágenes disponibles gratuitamente en Google Earth o Bing Maps tienen más de una década desde su adquisición, por lo que será necesaria una actualización periódica de nuestra base de datos, en especial si se pueden incorporar otras fuentes de imágenes satelitales. En este sentido, es alentador el desarrollo, aún incipiente, de plataformas como Planet (www.planet.com), que ofrecerán imágenes globales con alta resolución espacial y temporal a partir de una constelación de más de 140 microsatélites de observación terrestre. La información espectral contenida en este tipo de imágenes, junto con conjuntos de datos como los presentados en este trabajo, que permitan entrenar algoritmos de clasificación, constituyen la base para el desarrollo y aplicación de procesos automáticos para la detección de sitios impactados por el castor. 


\section{CONCLUSIONES}

Por primera vez a partir de un relevamiento exhaustivo se describieron de forma cuantitativa los patrones espaciales de la distribución y los impactos del castor, un ingeniero de los ecosistemas exótico e invasor, en la porción argentina de la Isla Grande de Tierra del Fuego. Este trabajo ejemplifica las ventajas de usar imágenes satelitales de alta resolución como una herramienta para estudiar, a escala regional, el impacto que generan las especies introducidas, ingenieras de los ecosistemas; en particular, en lugares remotos y de difícil acceso. Nuestro estudio aporta información relevante para manejar esta especie y brinda una base sólida para futuras investigaciones. Esta información, pública y de uso libre para fines científicos y de divulgación, permitirá evaluar los controles biofísicos y humanos de la invasión, monitorear la invasión en el espacio y en el tiempo, y habilitará la detección temprana de nuevas áreas invadidas.

Agradecimientos. A Alejandro Pietrek, que realizó valiosos aportes en aspectos clave del trabajo. Agradecemos también a Julio Escobar, Leonardo Collado y a Sobre La Tierra (http://sobrelatierra.agro.uba.ar). El editor asociado y dos revisores contribuyeron a mejorar el manuscrito. Este trabajo se realizó con recursos de la Facultad de Agronomía de la Universidad de Buenos Aires.

\section{REFERENCIAS}

Anderson, C. B., C. R. Griffith, A. D. Rosemond, R. Rozzi, and O. Dollenz. 2006. The effects of invasive North American beavers on riparian plant communities in Cape Horn, Chile: Do exotic beavers engineer differently in sub-Antarctic ecosystems? Biological Conservation 128:467-474.

Baker, B. W., and E. P. Hill. 2003. Beaver (Castor canadensis). Pp. 288-310 in G. A. Feldhamer, B. C. Thompson and J. A. Chapman (eds.). Wild Mammals of North America: Biology, Management and Conservation. The Johns Hopkins University Press, Baltimore, Maryland, USA.

Baldini, A., J. Oltremari, and M. Ramírez. 2008. Impacto del castor (Castor canadensis, Rodentia) en bosques de lenga (Nothofagus pumilio) de Tierra del Fuego, Chile. Bosque 29:162-169.

Bellard, C., P. Cassey, and T. M. Blackburn. 2016. Alien species as a driver of recent extinctions. Biology Letters 12: 06-23.

Briones, M., R. Schlatter, A. Wolodarsky, and C. Venegas. 2001. Clasificación ambiental para hábitat de Castor canadensis (Kuhl 1820, Rodentia), de acuerdo a características de cuencas en un sector de Tierra del Fuego. Anales del Instituto de la Patagonia 29:75-93.

Butler, D. 2006. Virtual globes: The web-wide world. Nature 439:776-778.

Butler, D. 2012. Characteristics of beaver ponds on deltas in a mountain environment. Earth Surface Processes and Landforms 37:876-882.

Chapin III, F. S., E. S. Zavaleta, V. T. Eviner, R. L. Naylor, P. M. Vitousek, H. L. Reynolds, and S. Díaz. 2000. Consequences of changing biodiversity. Nature 405:234-242.

Coronato, A., J. Escobar, C. Mallea, C. Roig, and M. Lizarralde. 2003. Características geomorfológicas de ríos de montaña colonizados por Castor canadensis en Tierra del Fuego, Argentina. Ecología Austral 13:15-26.

Courchamp, F., J. L Chapuis, and M. Pascal. 2003. Mammal invaders on islands: impact, control and control impact. Biological Reviews 78:347- 383.

Ehrenfeld, J. G. 2010. Ecosystem consequences of biological invasions. Annual review of ecology evolution and systematics 41:59-80.

Fretwell, P. T., M. A. LaRue, P. Morin, G. L. Kooyman, B. Wienecke, N. Ratcliffe, A. J. Fox, A. H. Fleming, C. Porter, and P. N. Trathan. 2012. An emperor penguin population estimate: the first global, synoptic survey of a species from space. PLoS ONE 7:e33751. DOI: 10.1371/journal.pone.0033751.

Graells, G., D. Corcoran, and J. C. Araven. 2015. Invasion of North American beaver (Castor canadensis) in the province of Magallanes, southern Chile: Comparison between dating sites through interviews with the local community and dendrochronology. Revista Chilena de Historia Natural 88:1-9.

Henn, J. J., C. B. Anderson, and G. Martínez Pastur. 2016. Landscape-level impact and habitat factors associated with invasive beaver distribution in Tierra del Fuego. Biological Invasions 18:1679-1688.

Hyvönen, T., and P. Nummi. 2008. Habitat dynamics of beaver Castor canadensis at two spatial scales. Wildlife Biology 14:302-308.

Iturraspe, R., and A. Urciuolo. 2000. Clasificación y caracterización de las cuencas hídricas de Tierra del Fuego. Actas del XVIII Congreso Nacional del Agua. Santiago del Estero, Argentina.

Johnston, C. A., and R. J. Naiman. 1990. The use of a geographic information system to analyze long-term landscape alteration by beaver. Landscape Ecology 4:5-19.

Jones, C. G., J. H. Lawton, and M. Shachak. 1994. Organisms as ecosystem engineers. Oikos 69:373-386.

Köppen, W. 1936. Das geographische System der Klimate. Pp. 1-44 in W. Köppen and R. Geiger (eds.). Handbuch der Klimatologie. Gebrüder Borntraeger, Berlin. 
Krebs, C. J. 1989. Ecological Methodology. Harper and Row. New York, USA.

Lizarralde, M. S. 1993. Current status of the introduced beaver (Castor canadensis) population in Tierra del Fuego, Argentina. Ambio 22:351-358.

Lizarralde, M., J. Escobar, and G. Deferrari. 2004. Invader species in Argentina: a review about the beaver (Castor canadensis) population situation on Tierra del Fuego ecosystem. Interciencia 29:352-356.

Lizarralde, M., J. Escobar, G. Deferrari, and M. Fasanella. 2008. El castor austral. Investigación y Ciencia 379:58-64.

Naiman, R. J., C. A. Johnston, and J. C. Kelley. 1988. Alteration of North American streams by beaver. BioScience 38: 753-762.

Mack, R. N., D. Simberloff, W. Mark Lonsdale, H. Evans, M. Clout, and F. A. Bazzaz. 2000. Biotic invasions: causes, epidemiology, global consequences and control. Ecological applications 10:689-710.

Malmierca, L., M. F. Menvielle, D. Ramadori, B. Saavedra, A. Saunders, N. Soto Volkart, and A. Schiavini. 2011. Eradication of beaver (Castor canadensis), an ecosystem engineer and threat to southern Patagonia. PP. 87-90 in C. Veitch, M. Clout and D. Towns (eds.). Island Invasives: Eradication and Management. International Conference on Island Invasives, IUCN (International Union for Conservation of Nature), Gland, Switzerland.

Martin, S. L., B. L. Jasinski, A. D. Kendall, T. A. Dahl, and D. W. Hyndman. 2015. Quantifying beaver dam dynamics and sediment retention using aerial imagery, habitat characteristics, and economic drivers. Landscape Ecology 30: 1129-1144.

Martínez Pastur, G., M. V. Lencinas, J. Escobar, P. Quiroga, L. Malmierca, and M. Lizarralde. 2006. Understorey succession in Nothofagus forests in Tierra del Fuego (Argentina) affected by Castor canadensis. Applied Vegetation Science 9:143-154.

Moore, D. M. 1983. Flora of Tierra del Fuego. Anthony Nelson - Missouri Botanical Garden, Londres.

Moorman, M. C., D. B. Eggleston, C. B Anderson, A. Mansilla, and P. Szejner. 2009. Implications of beaver Castor canadensis and trout introductions on native fish in the Cape Horn biosphere reserve, Chile. Transactions of the American Fisheries Society 138:306-313.

Morrison, A., C. J. Westbrook, and A. Bedard-Haughn. 2014. Distribution of Canadian rocky mountain wetlands impacted by beaver. Wetlands 35:95-104.

Pietrek, A. G., and L. Fasola. 2014. Origin and history of the beaver introduction in South America. Mastozoología Neotropical 21:355-359.

Pietrek, A. G., and M. González-Roglich. 2015. Post-establishment changes in habitat selection by an invasive species: beavers in the Patagonian steppe. Biological Invasions 17:3225-3235.

Pietrek, A. G., J. M. Escobar, L. Fasola, I. Roesler, and A. Schiavini. 2017. Why invasive Patagonian beavers thrive in unlikely habitats: a demographic perspective. Journal of Mammalogy 98:283-292.

Pisano, E. 1977. Fitogeografía de fuego-patagonia chilena. I comunidades vegetales entre las latitudes $52^{\circ}$ Y $56^{\circ} \mathrm{S}$. Anales Del Instituto de La Patagonia 8:121-250.

Schiavini, A., M. L. Carranza, G. Deferrari, J. Escobar, L. Malmierca, and A. G. Pietrek. 2016. Erradicación de especies invasoras: ciencia, actitud y entendimiento. El castor en tierra del fuego. Mastozoología Neotropical 23:279-288.

Skewes, O., F. González, R. Olave, A. Ávila, V. Vargas, P. Paulsen, and H. E. König. 2006. Abundance and distribution of American beaver, Castor canadensis (Kuhl 1820), in Tierra del Fuego and Navarino islands, Chile. European Journal of Wildlife Research 52:292-296.

SMN. 2018. Caracterización: Estadísticas de largo plazo. url: www.smn.gob.ar/caracterización-estadísticas-de-largoplazo.

Tershy, B. R., K. W. Shen, K. M. Newton, N. D. Holmes, and D. A. Croll. 2015. The importance of islands for the protection of biological and linguistic diversity. Bioscience 65:592-597.

Turetsky, M. R., and V. L. S. Louis. 2006. Disturbance in boreal peatlands. Pp. 359-379 in R. K. Wieder and D. H. Vitt (eds.). Boreal Peatland Ecosystems. Springer-Verlag, Berlin, Germany.

Valenzuela, A. E. J., C. B. Anderson, L. Fasola, and J. L. Cabello. 2014. Linking invasive exotic vertebrates and their ecosystem impacts in Tierra del Fuego to test theory and determine action. Oecologica 54:110-118.

Visser, V., B. Langdon, A. Pauchard, and D. M. Richardson. 2013. Unlocking the potential of Google Earth as a tool in invasion science. Biological Invasions 16:513-534.

Vitousek, P. M., C. D’Antonio, L. L. Loope, and R. Westbrooks. 1996. Biological invasions as global environmental change. American Scientist 84:468-78.

Wallem, P. K., C. G. Jones, P. A Marquet, and F. M. Jaksic. 2007. Identificación de los mecanismos subyacentes a la invasión de Castor canadensis (Rodentia) en el archipiélago de Tierra del Fuego, Chile. Revista Chilena de Historia Natural 80:309-325.

Wright J. P., C. G. Jones, and A. S. Flecker. 2002. An ecosystem engineer, the beaver, increases species richness at the landscape scale. Oecologia 132:96-101.

Yu, L., and P. Gong. 2012. Google Earth as a virtual globe tool for Earth science applications at the global scale: progress and perspectives. International Journal of Remote Sensing 33:3966-3986. 